SLAC-PUB-8651

BABAR-PROC-00/38

hep-ex/0010058

October 2000

\title{
PIN Photodiodes for Radiation Monitoring and Protection in the BABAR Silicon Vertex Tracker*
}

\author{
T.I. Meyer \\ Stanford Linear Accelerator Center, Stanford University, Stanford, CA 94309 \\ representing the BABAR Collaboration
}

\begin{abstract}
We discuss the design, implementation and performance of the radiation monitoring and protection system used by the Silicon Vertex Tracker (SVT) in the BaBar detector. Using 12 reverse-biased PIN photodiodes mounted around the beampipe near the IP, we are able to provide instantaneous radiation dose rates, absorbed dose integrals, and active protection that aborts the circulating beams in the PEP-II storage ring when radiation levels exceed user-defined thresholds. The system has reliably protected the SVT from excessive radiation damage and has also served as a key diagnostic tool in understanding radiation backgrounds at PEP-II.
\end{abstract}

\author{
(Contributed to) \\ The Meeting of \\ The Division of Particle and Fields \\ of The American Physical Society \\ Columbus, Ohio, USA \\ August 9-August 12, 2000
}

${ }^{*}$ Work supported by Department of Energy contract DE-AC03-76SF00515. 


\section{Motivation}

The unprecedented beam currents and luminosities at the PEP-II accelerator make machine backgrounds a significant challenge to the BaBar experiment. Despite significant use of radiation hard technology, the Silicon Vertex Tracker (SVT) is the most radiation vulnerable sub-detector because of its proximity to the beamline. The SVT has established a rigorous program of radiation monitoring and protection (SVTRAD) to ensure the proposed lifespan, including the ability to automatically abort the beams in PEP-II when radiation exceeds programmable thresholds ${ }^{1}$.

\section{Design}

Reverse-biased large-area PIN photodiodes (Hamamatsu S3590-08) were selected to meet the multiple constraints of space, expense, and scalability. Signal current is generated from the deposition of energy from incident radiation that creates electron-hole pairs which flow under the applied field. (200 pC $\sim 1$ mRad of absorbed dose) By continuously accumulating the the instantaneous dose rates, absorbed dose integrals can be formed with well-controlled fractional errors. Precision signal extraction depends largely on knowledge of the pedestal, which depends on temperature and absorbed dose (through radiation damage).

The SVTRAD system uses 2 rings of 6 radiation sensors at $\mathrm{z}= \pm 20 \mathrm{~cm}$ from the inter-

action point at a radius of $3 \mathrm{~cm}$. Each photodiode is accompanied by 2 closely neighboring thermistors. Shielded signal cables carry the diode currents and bias voltage from the sensors to the front-end electronics module within the SVT shielding scheme.

\section{Implementation}

The SVTRAD module is designed to be autonomous and robust ${ }^{2}$. It maintains configuration information between power-on resets and internally buffers messages for remote client retrieval. Each channel in the SVTRAD module can be configured to be used either for radiation monitoring or protection. Channels used in the protection circuit are passed back to the monitoring path, but resolution is degraded.

Each SVTRAD module handles three channels of current input. The monitoring circuit uses a set of three charge-integrating 20-bit Burr-Brown DDC101 ADCs, two sets of memory buffers, and a Xilinx 4013E FPGA. The ADCs are configured to average 4 on-chip $0.25 \mathrm{~ms}$ conversions to deliver data at $1 \mathrm{kHz}$. This data is split into two streams ("slow" and "fast") which are directed into separate registers. The slow datastream is internally accumulated within the FPGA and rolled out to fill the $8 \mathrm{~K}$ memory at $0.47 \mathrm{~Hz}$. The fast memories implement a circular history buffer with two back-to-back $32 \mathrm{~K}$ buffers, which can be read out at remote request. The fast history buffer provides an average history of 5.7 seconds at $1 \mathrm{kHz}$ sampling.

The primary radiation concern for the SVT is integrated dose, and therefore the radiation protection algorithm focuses on placing a threshold on a minimum (integrated) dose absorbed 
over a minimum time. The algorithm enforces a chronic dose rate threshold (i.e. $\mathrm{mR} / \mathrm{s}$ ), but with a dose tolerance measured in $\mathrm{mR}$. The tolerance parameter sets how much dose is integrated above the chronic dose rate threshold before the circuit trips. Short term departures over the chronic dose rate threshold are therefore allowed, so long as their integral is less than the tolerance.

Implementation uses a set of Analog Devices AD652s (so-called current-to-frequency converters) and an Orca 2C26A FPGA. The FPGA maintains a multi-input counter, whose inputs are the converted diode frequency, an internal programmable timer, and the thermistor current converted frequency. The diode and thermistor frequencies are oppositely signed and the programmable timer rate is chosen to enforce the desired threshold. When the count value exceeds the (programmable) depth of the counter, the circuit trips.

\section{Performance}

The SVTRAD module is supported over a local Controller Area Network by an application built using the EPICS toolkit. The EPICS database provides controls, monitoring processes, and client connections over TCP/IP for displays and archiving. The data retrieved from the SVTRAD module is processed by a set of custom EPICS records and subroutines The ADC samples are combined with temperature measurements from other modules in the common EPICS environment to form average dose rate measurements.

Performance of the SVTRAD system is best illustrated in Figure 1(a), showing the evolution of integrated doses for several of the diodes. In 1999 alone, there were over 4500 beam aborts caused by SVTRAD, and another 2500 in the year 2000 (see Figure 1(b)). Reviewing the fast history buffer after a trip has been an invaluable tool, providing validation of the protection thresholds.

Systematic errors from pedestal imprecision contribute less than $10 \%$ absolute error to the absorbed dose integrals, and instantaneous dose rates are measured with better than $0.25 \mathrm{mR} / \mathrm{s}$ accuracy in the monitoring circuit, and $5 \mathrm{mR} / \mathrm{s}$ for the protection diodes. Trip thresholds are accurate to $10 \mathrm{mR} / \mathrm{s}$. These performance values will degrade as integrated radiation damage exceeds $1 \mathrm{MRad}$, however.

The radiation budget of the SVT must be measured against the constraints it places on general data-taking. The trip thresholds were optimized for minimizing overall radiation exposure to the SVT while guaranteeing headroom for the accelerator to reach their performance goals.

The SVTRAD system has proven effective in limiting radiation exposure of the SVT. The online dose rate information is used extensively by background remediation experts to identify background sources and locations ${ }^{3}$.

\section{Acknowledgments}

We thank the PEP-II accelerator physicists and operators for many valuable discussions. 

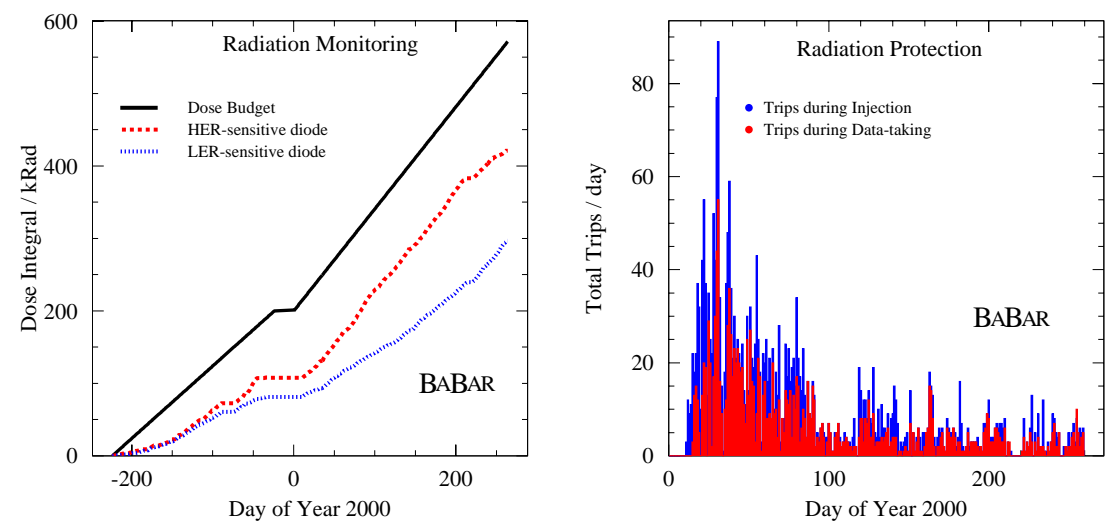

Figure 1: (a) Integrated dose history for several of the diode sensors, compared to the 1999-2000 SVT radiation budget. (b) By actively aborting beams when radiation exceeds thresholds, the SVTRAD system has successfully protected the SVT.

\section{References}

[1] BaBar Collaboration, Technical Design Report (1995).

[2] T.I. Meyer, http://hep.stanford.edu/babar/monitoring/svtrad/docs.html.

[3] T.I. Meyer, http://www.slac.stanford.edu/ meyertim/svtrad/bkgs/. 